\title{
Overlap of isochrone resonances: Chaos and refraction
}

\author{
R. Egydio de Carvalho \\ Instituto de Geociências e Ciências Exatas, Universidade Extadual Paulista-UNESP, 13500-230 Rio Claro, SP, Brazil
}

(Received 24 June 1996)

\begin{abstract}
We consider the two nonconcentric circles billiard, with the inner circle as a refringent medium, in order to study the classical dynamics of a light ray. The eccentricity controls the chaotic sea intensity and the refraction index acts on the integrable portion of the phase space, prompting the appearance and overlapping of isochrone resonances. Numerical results are presented and discussed. [S1063-651X(97)08602-9]
\end{abstract}

PACS number(s): 42.15.-i, 03.20. $+\mathrm{i}, 46.90 .+\mathrm{s}$

In classical dynamics, resonances are usually accompanied by a chaotic layer and the overlap connecting the layers amplify the regions occupied by chaos. On the other hand, classical resonance interactions in integrable (local or global) contexts have been reported during the last few years and referred to as reconnection process or overlap of isochrone resonances. This corresponds to a topological rearrangement of the phase space structures where the island chains interact among themselves without introducing chaos. If the chains are in phase, the hyperbolic points from different chains coalesce, but most frequently there is a phase shift among them such that during the overlap process, the manifolds change their original path and go into other separatrix regions. This phenomenon has been reported in studies of the radial twist mapping in a nonintegrable scenario [1]. The Hénon map and nontwist maps have been utilized to analyze the reconnection of dimerized chains [2]. In another approach, a Hamiltonian is derived using the Birkhoff-Gustavson normal form around an elliptic fixed point [3] to obtain an $n$-order polynomial in one action variable, yielding up to $(n-1)$ island chains which may overlap in a globally integrable context. Numerical simulations of confined plasma in tokamaks exhibit magnetic island chains and integrable overlap is also reported [4]. Stochastic webs are examples of in phase island chain interaction [5]. Such resonances have also been noticed in beam-beam interaction dynamics [6]. The purpose of this work is to study the classical dynamics of a light beam, using a billiard model, in the context of a chaos and isochrone resonance scenario.

Our billiard model is shaped by two eccentric circles in the $(x, y)$ plane where the outer circle has radius $R=1$, corresponding to an infinite barrier, while the inner circle has radius $r$ and it corresponds to a refringent medium with a variable refraction index, denoted by $n$. The intermediate region between both circles has refraction index $n=1$. The inner circle may dislocate horizontally with respect to the center of the outer circle, and the distance $\delta$ between the centers is defined as the eccentricity. Although $r$ and $\delta$ may assume different values, they are limited by the constraint $(r+\delta) \leqslant 1$, thus there are two free parameters $n$ and $\delta$ playing the role of perturbation parameters which may introduce interesting effects into the dynamics. The billiard trajectories move in broken lines between collisions with the external boundary. It is convenient to use Birkhoff's area preserving mapping coordinates $(L, S)[7]$ to obtain the mapping equations, where $L$ is the $2 \pi$-normalized arc length around the outer circle and $S$ is the sine of the reflection angle $\alpha$ at every collision with the outer circle, so that $-\frac{1}{2} \leqslant L \leqslant \frac{1}{2}$ and $-1 \leqslant S \leqslant 1$. The mapping equations connect $\left(\theta_{i}, \alpha_{i}\right)$ with $\left(\theta_{i-1}, \alpha_{i-1}\right)$, where $\theta$ is the angle related to the arc length $L=(\theta / 2 \pi)$, preserving the area $\iint \sin \alpha d \alpha d \theta$. There are two kinds of motion, labeled here as $A$ and $B$. The $A$ motion corresponds to those orbits which hit the outer circle after a collision with the same circle and the $B$ motion corresponds to those orbits which attain the inner circle between two collisions with the outer one. There is also a particular family of orbits which never attain the inner circle, called whispering gallery orbits (WGO). The latter are identified as the orbits of nonzero measure, which never cross the caustics (an auxiliary circle of radius $r+\delta$ ).

We consider a monochromatic light ray traveling inside the two circles billiard which totally reflects at the outer circle, and we assume the ideal limit of total refraction at the inner circle. The reflection law is as follows: incidence angle equals reflection angle, while the refraction obeys Snell's law: $n_{1} \sin \beta_{1}=n_{2} \sin \beta_{2}$. Taking $\beta_{1}$ and $\beta_{2}$ to be the angles between the trajectory and the normal to the inner circle, we see that if $n_{2}>n_{1}$ then $\beta_{2}<\beta_{1}$ for $0 \leqslant\left|\beta_{i}\right| \leqslant \pi / 2$. Thus if the trajectory goes to a more refringent medium it deviates closer to the normal, and reciprocally, it deviates away from the normal when it goes to a medium with smaller refraction

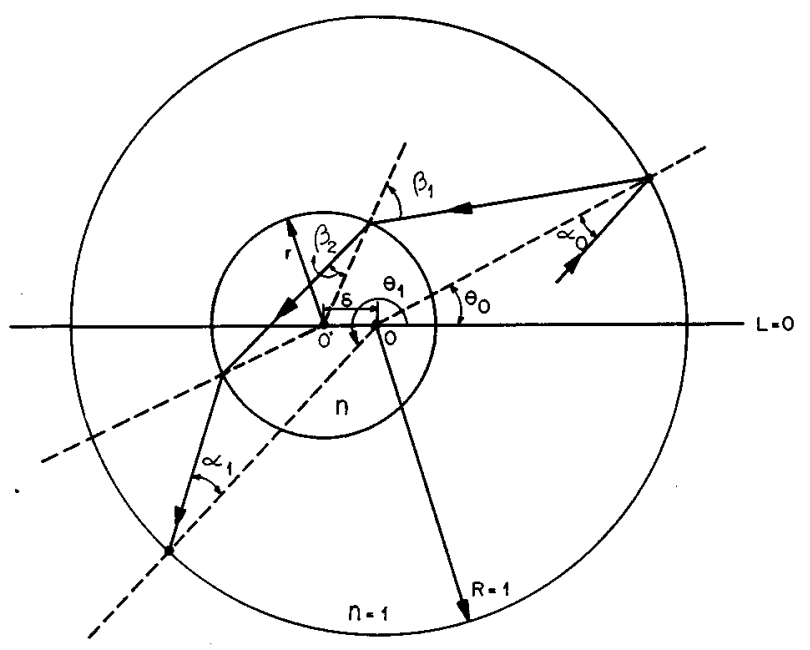

FIG. 1. A geometrical scheme of the light beam motion in the billiard model. 


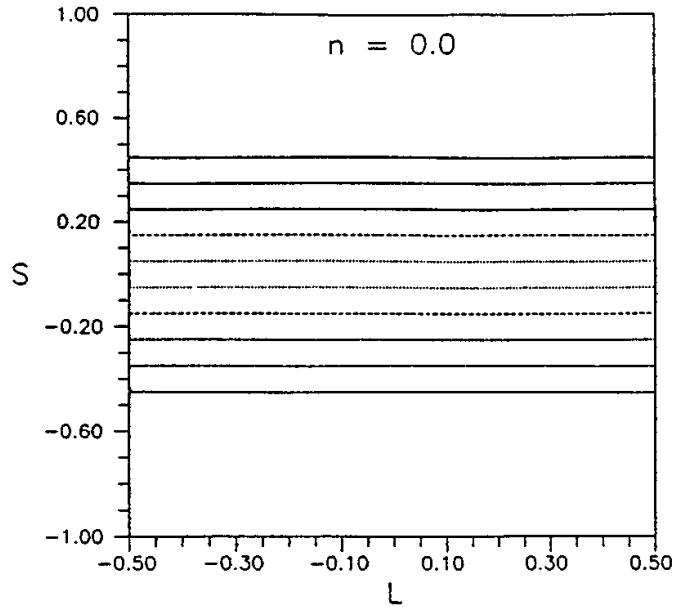

(o)

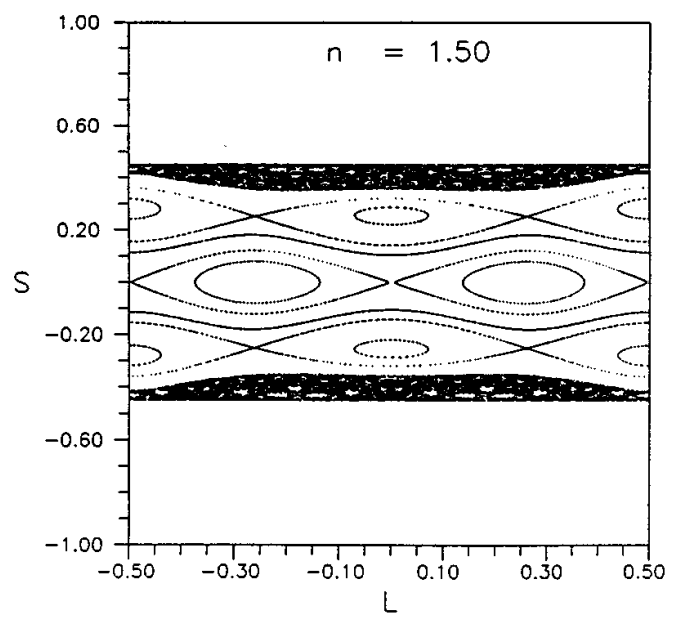

(c)

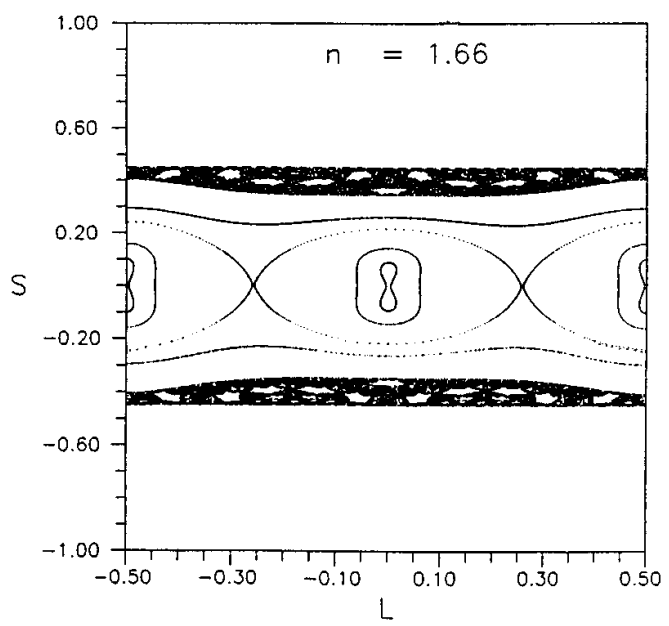

(e)

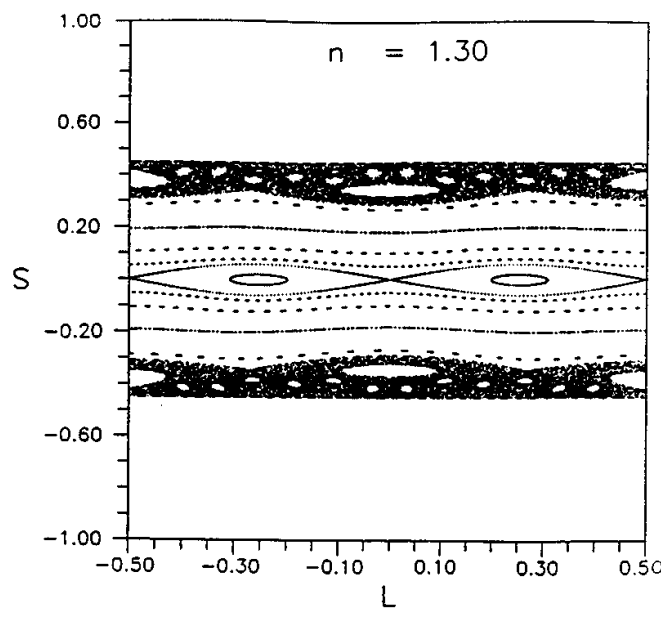

(b)

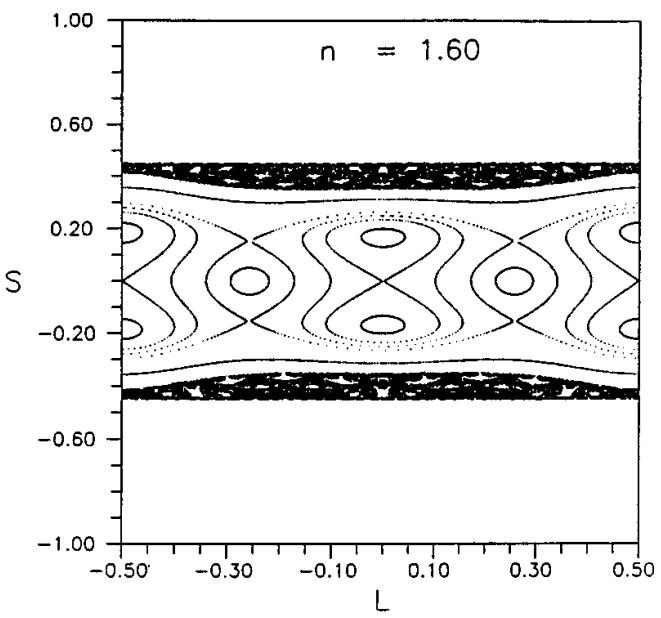

(d)

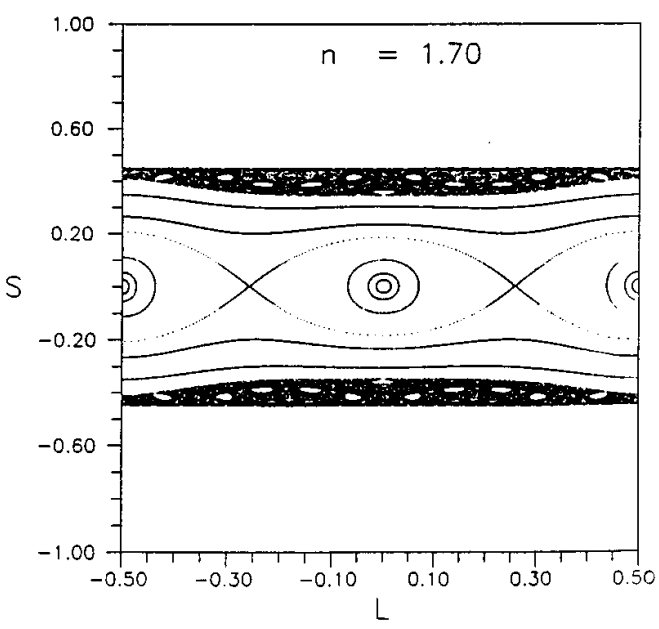

(f)

FIG. 2. The effect of varying the refraction index $n$ with $r=0.40$ and $\delta=0.05$ is analyzed for (a) $n=0.0$, the completely integrable case; (b) $n=1.30$, there are tori, an island chain, and two symmetrical chaotic regions with islands; (c) $n=1.50$, three chains are in the regular region of the phase space; (d) $n=1.60$, the overlap connecting the three chains has already set in; (e) $n=1.66$, the first saddle-center bifurcation; and (f) the pendulumlike final configuration, after the second saddle-center bifurcation.

index. From Fig. 1, considering an initial condition $\left(\alpha_{0}, \theta_{0}\right)$, we derive the analytical mapping equations for the $B$ motion:

$$
\sin \alpha_{1}=r \sin \beta_{1}+\delta \sin u,
$$

$$
\theta_{1}=2 \pi-\alpha_{1}-u
$$

with

$$
u=\pi-2 \beta_{1}+2 \beta_{2}-\theta_{0}+\alpha_{0}
$$




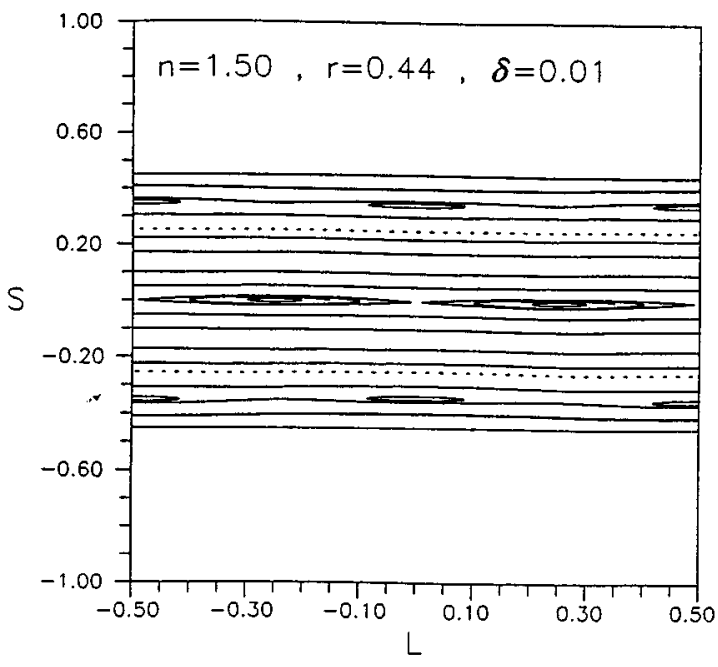

(a)

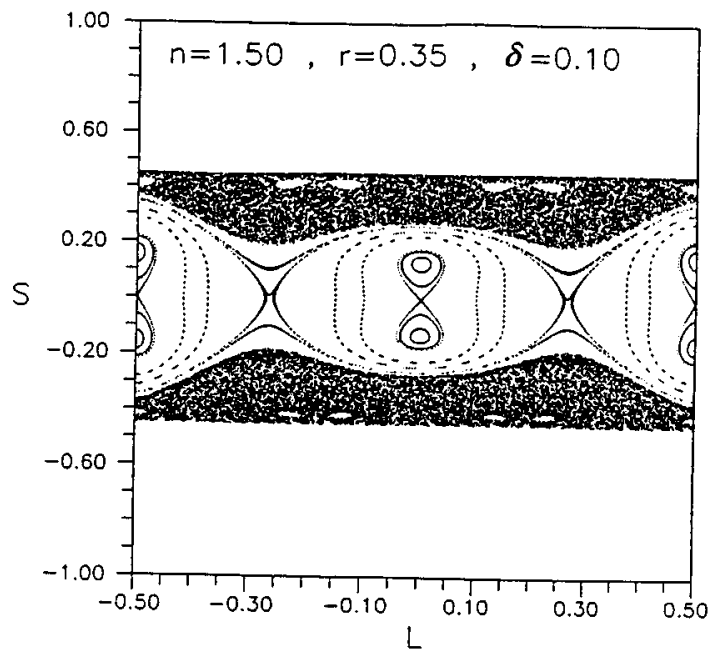

(b)

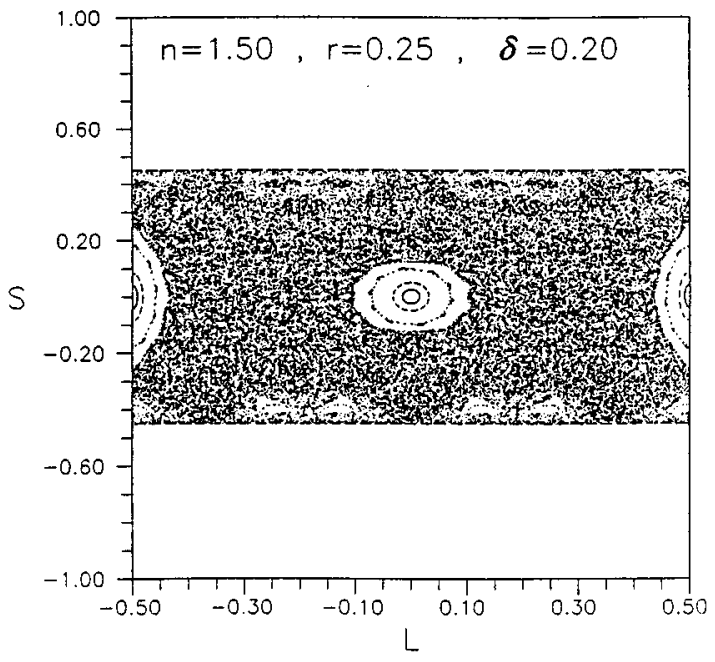

(c)

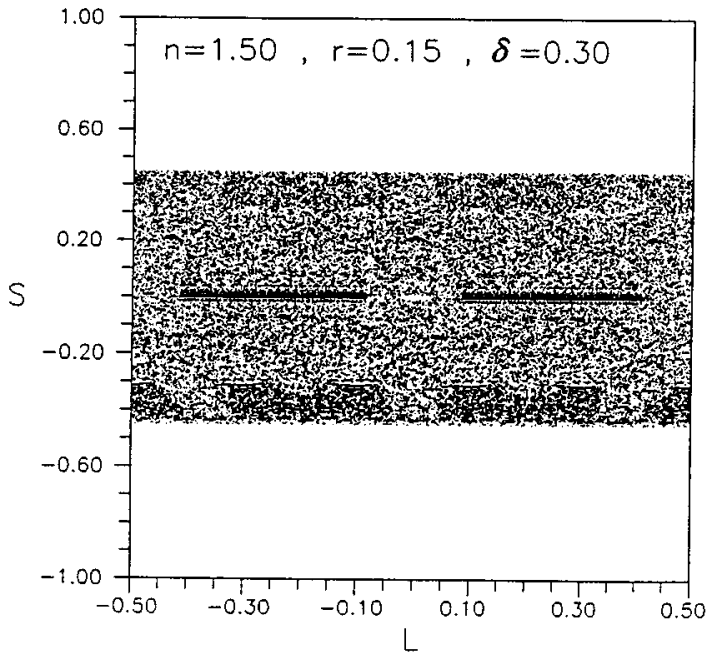

(d)

FIG. 3. The effect of varying the eccentricity with $(r+\delta)=0.45$ and $n=1.50$ is analyzed for (a) $\delta=0.01$, three thin island chains are present [for smaller values of $\delta$, the phase space would tend to be like Fig. 2(a)]; (b) $\delta=0.10$, we see some structures and the two symmetrical chaotic regions; (c) $\delta=0.20$, chaos has increased; and (d) $\delta=0.30$, the phase space is almost fully chaotic.

and

$$
\beta_{2}=\sin ^{-1}\left[\frac{1}{n} \sin \beta_{1}\right] .
$$

The incidence angle $\beta_{1}$ at the refracting circle is obtained by considering this circle as an infinite reflecting barrier where the trajectories would be elastically reflected. This scheme yields the known formula [7]

$$
\sin \beta_{1}=\frac{1}{r}\left[\sin \alpha_{0}+\delta \sin \left(\alpha_{0}-\theta_{0}\right] .\right.
$$

Hence the mapping given by Eqs. (1) and (2) is well defined and the $A$ motion is simply given by

$$
\begin{gathered}
\alpha_{1}=\alpha_{0}, \\
\theta_{1}=\theta_{0}+\left(\pi-2 \alpha_{0}\right) .
\end{gathered}
$$

The dynamical analysis has two aspects. First, we observe the effect of varying the refraction index $n$ keeping constant $r=0.40$ and $\delta=0.05$, see Figs. 2(a) $-2(\mathrm{f})$; and second, we observe the effect of the eccentricity $\delta$ for $n=1.50$ and $(r$ $+\delta)=0.45$, see Figs. 3(a) $-3(\mathrm{~d})$. In the first case, we see that for $n=0.0$, the refringent medium does not exist and the system is completely integrable. For $n=1.30$, there is a well 
formed thin chaotic layer near the values $|S|=0.45$ and an island chain near $S=0.0$; for $n=1.50$, we see two more island chains in the regular portion of the phase space; for $n=1.60$, we observe the three chains overlapping in a local integrable context; for $n=1.66$ the first elliptic-saddle point bifurcation has already occurred; for $n=1.70$, the second bifurcation has taken place. For greater values of $n$, the integrable region between the chaotic layers is similar to the pendulum phase space. These plots show that the refraction index does not introduce chaos, nor does it affect the present chaotic sea. On the contrary, it acts only on the resonant integrable region and it is responsible for the resonances overlapping. The clear regions, i.e., $|S|>0.45$, correspond to the WGO tori, which are simply straight lines. In the second case, $n=1.50$, we initially choose $(r, \delta)=(0.44,0.01)$, a near integrable situation, where thin islands may be observed among the tori; for $(r, \delta)=(0.35,0.10)$ we see some structures and chaos; for $(r, \delta)=(0.25,0.20)$ the chaotic sea increased and for $(r, \delta)=(0.15,0.30)$ chaos almost occupies the full accessible region. So, we conclude that the eccentricity is responsible for the chaos intensity. Hence we have two distinct effects: overlap of isochrone resonances due to the variable refraction index and chaos due to the eccentricity; each effect driven by one parameter. It is also possible to establish a two dimensional mechanical analogy to this problem, regarding energy and momentum conservation, by considering the inner circle as a finite potential $V_{0}$, in such a way that if the particle has energy smaller than $V_{0}$, it is totally reflected. On the other hand, considering nontrapped motion [8], it suffers a deviation like a refraction process, if its energy is higher than $V_{0}$.

The occurrence of isochrone integrable resonances overlapping in one more approach, the refraction of a light beam, suggests that we consider it as a more fundamental physical manifestation, and the transition to chaos in an optical medium with different refraction indices, using the two circle billiard model, may be relevant to some aspects of propagation of light in optical fibers.

I thank S. P. Carvalho and S. M. O. K. L. da Silva for fruitful discussions and the scientific Brazilian agencies FAPESP, CNPq, and FUNDUNESP for financial support.
[1] J. E. Howard and S. M. Hohs, Phys. Rev. A 29, 418 (1984).

[2] J. P. Van Der Wheele and T. P. Valkering, Physica A 169, 42 (1990); 153, 283 (1988).

[3] R. Egydio de Carvalho and A. M. Ozório de Almeida, Phys. Lett. A 162, 457 (1992); R. Egydio de Carvalho, Nonlinearity 6, 973 (1993).

[4] G. Oda and I. L. Caldas, Chaos Solitons Fractals 5, 15 (1995).

[5] A. A. Chernikov, R. Z. Sagdeev, and G. M. Zaslavsky, Physica D 33, 65 (1988).
[6] A. Gerasimov, F. M. Izrailev, J. L. Tennyson, and A. B. Temnykh, in Nonlinear Dynamics Aspects of Particle Accelerators, edited by J. M. Jowett, M. Month, and S. Turner, Springer Lecture Notes in Physics Vol. 247 (Springer, New York, 1986), p. 154; reprinted in Hamiltonian Dynamical Systems, edited by R. S. Mackay and J. D. Meiss (Hilger, Bristol, 1987).

[7] O. Bohigas, D. Boose, R. Egydio de Carvalho, and V. Marvulle, Nucl. Phys. A560, 197 (1993).

[8] A. J. Fendrik, A. M. F. Rivas, and M. J. Sánchez, Phys. Rev. E 50, 1948 (1994). 